This large-scale analysis led to the identification of monogenic causes of lupus in about $7 \%$ of analysed patients in an unselected paediatric population of jSLE. 7/8 causes are related to an innate immune disorder with efferocytosis deficiency, emphasising the importance of apoptotic body clearance in the pathogenesis of lupus. Other variants in KLC or PLC genes may represent novel monogenic causes of lupus or could influence disease-onset by increasing the penetrance of more severe mutations. The treatment is still poorly adapted to the underlying mechanisms but progress in immunomonitoring together with the revolution in the field of genetics prompt clinician to set up targeted therapies considering genetic background and biomarkers.

Disclosure of Interest: A. Belot Grant/research support from: MERCK DOI: 10.1136/annrheumdis-2018-eular.7857

\section{SP0128 2 FINDING THE NEEDLE IN THE HAYSTACK AND USING IT: GALECTIN-9 AS A BIOMARKER IN JUVENILE DERMATOMYOSITIS}

A. Van Royen-Kerkhof. Pediatric Immunology and Rheumatology, Wilhelmina children's Hospital of the University Medical Center Utrecht, Utrecht, Netherlands

Background: Juvenile dermatomyositis (JDM) is a rare systemic immune-mediated disease involving skin and muscle. A high disease burden exists with risk of both under- and overtreatment due to the lack of reliable biomarkers.

Methods: A multiplex immunoassay was performed in a discovery cohort for plasma levels of 45 proteins related to inflammation in 25 well-defined JDM patients, determined by clinical activity and treatment.

Results were validated in two independent international external and internal validation cohorts $(n=125)$.

In a longitudinal cohort $(n=30)$, the performance of this biomarker over time was assessed with a median 2.8 years follow-up.

Results: In the discovery cohort we found a clustering of 10 mediators of which Galectin-9 and CXCL10 distinguished best between active disease and remission. Both biomarkers had a strong correlation with clinical parameters (Spearman $r$ with Physician's global assessment (PGA) $=0.75$ for both). This was confirmed in the validation cohorts (Spearman $r=0.7$ with $P G A$, for both). In the longitudinal cohort galectin-9 and CXCL10 correlated with disease activity over time, and elevated levels could predict flares several months before clinical symptoms. Both cross-sectionally and longitudinally, galectin-9 and CXCL10 outperformed creatine kinase activity.

Conclusion: Galectin- 9 and CXCL10 are robust biomarkers for disease activity in JDM. A short-term implementation into clinical practice is feasible and can facilitate individualised treatment.

Disclosure of Interest: None declared

DOI: 10.1136/annrheumdis-2018-eular.7847

FRIDAY, 15 JUNE 2018

\section{Triple T: T cells, technologies and therapies}

\section{SP0129 STUDYING T CELL FUNCTION IN RA AND PSA}

L. Taams. Inflammation Biology, King's College London, London, UK

Rheumatoid arthritis $(\mathrm{RA})$ and spondyloarthritis $(\mathrm{SpA})$ describe a group of inflammatory joint diseases affecting $\sim 2 \%$ of the population. RA has strong genetic associations with $H L A-D R$, indicating a role for CD4 $+\mathrm{T}$ cells. CD $4+\mathrm{T}$ cells are prominently present in the RA joint, where they can contribute to the inflammatory milieu. I will present recent data from our lab regarding the presence, regulation and function of different CD4 +T cell subsets that are present in the RA joint.

In contrast, SpA has strong genetic associations with $H L A-B / R U N X 3$ which imply a role for $\mathrm{CD} 8+T$ cells. Furthermore, genetic associations with IL23R/TRAF3IP2 and the clinical efficacy of IL-17 blockade in SpA, indicate a role for IL-17 in SpA. This provides a strong rationale to investigate the presence, phenotype and functional capacity of IL-17 +CD8+T cells in the joints of patients with SpA. I will present novel data regarding the presence, phenotype and potential function of $\mathrm{IL}-17+\mathrm{CD} 8+\mathrm{T}$ cells in the joints of patients with SpA. Collectively, our data indicate that IL-17+CD8+T cells may be important contributors to the pathogenesis of $\mathrm{SpA}$

Disclosure of Interest: L. Taams Grant/research support from: GSK, UCB, Novo Nordisk A/S, Novartis

DOI: 10.1136/annrheumdis-2018-eular.7808

\section{SP0130 TOWARDS T CELL TOLERANCE IN RHEUMATOID ARTHRITIS}

\section{R. Thomas. Diamantina Institute, University of Queensland, Brisbane, Australia}

Disease modifying strategies are available for treatment of rheumatoid arthritis (RA), and good response rates are achieved. However, limitations include toxicity, a response rate ceiling, cost and rationing of biologic therapies, inability to cure or permanently reverse RA pathology, and inability to prevent disease. Immuno therapies targeting checkpoint molecules are markedly changing the landscape of clinical oncology. In autoimmune diseases such as RA, dendritic cells represent an important target for antigen-specific immunotherapy for $\mathrm{T}$ cell tolerance. Antigen-specific strategies promise greater specificity and safety, without genera immune suppression, and thus the potential for intervention in at-risk subjects before disease onset. In a proof-of-concept trial, delivery of autoantigenic peptides and autologous tolerogenic dendritic cells was safe and had immunomodulatory effects on $T$ cells including reduction of effector $T$ cells and a relative increase in regulatory $T$ cells. We have developed and are trialling in RA patients, antigenspecific immunotherapy targeting dendritic cells in situ with liposomes encapsulating autoantigenic peptide and calcitriol with the aim of antigen-specific T cell tolerance. I will discuss the development of antigen-specific tolerance strategies and the parallel development of immune monitoring assays to determine $T$ cell outcomes in clinical trials in RA.

Disclosure of Interest: R. Thomas Grant/research support from: Janssen Biotech Inc, Consultant for: Janssen Biotech Inc

DOI: 10.1136/annrheumdis-2018-eular.7793

\section{FRIDAY, 15 JUNE 2018}

\section{Navigating the world of digital health}

\section{SP0131 ONLINE SOCIAL MEDIA PLATFORMS AND PUBLIC HEALTH INFORMATION: AN EXPLORATION INTO ARTHRITIS RELATED VIDEOS ON YOUTUBE IN 2017}

\section{E. Heron. The University of Southampton, Southampton, UK}

Background: YouTube is one of the most used social media platforms from a desktop computer. YouTube provides a virtual platform that allows users to upload and view video content. Due to this functionality, YouTube is a valuable method for sharing and disseminating health information.

Patients with chronic conditions (including arthritis) are increasingly relying on online health information to help manage their symptoms, with $75 \%$ of patients living with chronic conditions reporting their healthcare decisions are influenced by information found online.

To date there have been no studies that have explored the information available for people searching for advice and support about arthritis on YouTube.

Methods: Patient and public involvement (PPI) representatives contributed to defining terms likely to be used by members of the public with arthritis searching for self-management strategies on YouTube. These included "joint pain", "knee pain", "hip pain", "hand pain" AND "helping" or "improving". From each of these search terms the top 10 videos sorted by view counts were chosen. Videos were included if the content was related to arthritis, in English and published in 2017 Videos were excluded if they were inappropriate or offensive, not related to humans or duplicates. The top 50 videos sorted by view count were included for analysis. This data set was systematically coded by the team lead $(E H)$ and cross checked by an additional team member (AC). Coded data were analysed using SPSS.

Results: Eighty videos were retrieved, 7 videos were irrelevant, 9 were duplicates and 11 were non-English language videos. Sixty-three videos were included for analysis. From the top fifty videos (sorted by view count), "Herbal Medicine" $(n=14 ; 28 \%)$ was the most common category, followed by "Exercise and Stretching" $(n=12 ; 24 \%)$. The most watched video relating to the self-management of arthritis was related to "Herbal Medicine" with a view count close to two million $(n=1,930,905)$ within the four months since it had been posted online. Twenty five $(69 \%)$ of the arthritis management related videos originated from the USA, with the UK producing only one video. Fifteen (30\%) of the videos had been posted by self-reported health professionals. Nineteen $(38 \%)$ of the videos were commercial. None of the videos produced links to research to back up their claims. Conclusion: Sharing of health information on YouTube is unregulated. The most accessed videos include alternative approaches to self-management and are not posted by registered health care professionals (HCPs). Whilst a wide range of arthritis-related videos were retrieved, few were created by HCPs or reputable health care organisations. YouTube is a powerful tool for people with arthritis to 
access and use health information. Current findings demonstrate scope for HCPs and established healthcare organisations to further utilise YouTube for the dissemination of quality-controlled, evidence-based information.

Disclosure of Interest: None declared

DOI: 10.1136/annrheumdis-2018-eular.7751

\section{SP0132 MEASURING DIGITAL HEALTH LITERACY, WHY AND HOW?}

C. Drossaert. Psychology Health and Technology, University of Twente, Enschede, Netherlands

Digital health literacy or eHealth Literacy refers to a person's ability to search, select, appraise and apply online health information or appropriately use digital health applications. In this presentation I will address the issue of measurement of these skills. First, we will explore why it is important to measure digital health literacy and discuss the different aims of measuring. Second, we will address some of the currently available instruments, including the oldest and most used instrument, the eHealth Literacy Scale or EHEALS Norman \& Skinner, 2006 and some more recent instruments, including the eHealth Literacy Questionnaire, eHLQ Kayser et al. 2018 and the Digital Health Literacy Instrument, DHLI. Van der Vaart \& Drossaert, 2017. Of each instrument, I will briefly discuss its underlying theory, some empirical findings, and its strengths and weaknesses. I will conclude with discussing some general challenges in measuring digital health literacy and directions for future research.

Disclosure of Interest: None declared

DOI: 10.1136/annrheumdis-2018-eular.7798

\section{SP0133 PATIENT EXPERIENCES FROM A TELE-HEALTH INTERVENTION ON DISEASE ACTIVITY IN RA: THE KEEN AND THE RELUCTANT PATIENT}

L.R. Knudsen. Department of Rheumatology, Aarhus University Hospital, Aarhus, Denmark

Background: Recently, the effectiveness of monitoring disease activity in rheumatoid arthritis (RA) through a patient-reported outcome (PRO)-based tele-health follow-up strategy was compared to usual outpatient follow-up in the TeRA trial. Telemedicine interventions require patients taking an active role in the disease course and treatment, and assuming more responsibility for monitoring and identifying signs and symptoms of disease activity. ${ }^{2-3}$ The TeRA study examines the effectiveness of tele-health follow-up, but provides no insight into how patients experience this new approach to disease control.

Objectives: To explore the experiences of a PRO-based tele-health follow-up from the perspective of patients with RA and their experiences of increasing their active role and responsibility for disease control in particular.

Methods: Adopting a strategy of interpretive description, we conducted individual, semi-structured interviews with 15 RA patients participating in the tele-health follow-up. Participants were selected purposively and consecutive from both genders and with various ages, disease durations and disease severity. The analysis was inductive with a constant comparative approach. First, we identified the main themes conveying the participants' experiences. Then, we constructed patient typologies to explain different perspectives on the tele-health follow-up.

Results: Five themes covered the participants' experiences: 'A flexible solution', 'Responsibility','Knowledge of RA', 'Communication and involvement' and 'Continuity'. Two typologies: 'the keen patient' and 'the reluctant patient' represented opposite perspectives and preferences regarding the core value of and approach to the tele-health follow-up.

Conclusions: The participants had positive perceptions of the PRO-based telehealth follow-up and saw it as a flexible and resource-saving solution that can reduce the burden of unnecessary interruptions in everyday life. They reported disadvantages related to missing face-to-face contact with health professionals.

The two typologies, 'the keen' and 'the reluctant' patient, help us understand the patients' different needs, wishes and abilities to take part in tele-health follow-up. Our findings reveal a need for more insight into how tele-health follow-up could be integrated in routine clinical practice, paying special attention to how reluctant patients may be supported.

\section{REFERENCES}

[1] Thurah A, Stengaard-Pedersen K, Axelsen M, Fredberg U, Schougaard LMV, Hjollund NHI, et al. A tele-health follow-up strategy for tight control of disease activity in rheumatoid arthritis: results of the non-inferiority randomised controlled trial (the TeRA study). Arthritis Care Res 2017 [Epub ahead of print]
[2] Wildevuur SE, Simonse LW. Information and communication technologyenabled person-centred care for the "big five" chronic conditions: Scoping review. J Med Internet Res 2015;17:77

[3] Paré G, Mogadem K, Pineau G, St-Hilaire C. Clinical effects of home telemonitoring in the context of diabetes, asthma, heart failure and hyperten sion: A systematic review. J Med Internet Res 2010;12:21.

[4] Thorne S. Interpretive description: Qualitative Research for Applied Practice. New York and London: Routledge; 2016 (2nd ed.).

Disclosure of Interest: None declared DOI: 10.1136/annrheumdis-2018-eular.3066

FRIDAY, 15 JUNE 2018

\section{The rheumatologist-orthopaedic surgeon connexion in secondary fracture prevention}

\section{SP0134 THE CONNEXION BETWEEN FRACTURE CARE AND SECONDARY FRACTURE PREVENTION}

K. Åkesson. Dept Clinical Sciences Malmö, Lund University, Malmo, Sweden

That fracture begets fracture, is today an acknowledged reality. Nevertheless there are still gaps in fracture care and many are far from reaching the optimal treatment pathway. Ultimately, each fracture should be appropriately managed from the moment of the fracture, through the acute management of the patient and the fracture, through to rehabilitation and secondary prevention. Optimisation of every step leads to better post-fracture functioning and quality of life, in addition to a reduced risk of new fracture events where the next fracture often is more severe than the previous.

Fracture treatment has improved with newer implants (plates, screws, nails, joint replacements etc) being developed specifically for fragile bone. Standardising procedures and checklists have made a difference in reducing complications. In the field of anaesthesia advances allows for surgery in the increasingly frail older person with acceptable perioperative risks and outcome. Similarly, tailored medical management is essential also to improve rapid post-operative recovery. The team approach to prompt regaining of function and rehabilitation allows for faster return to the home.

However, it has been more difficult to systematically improve the final step - prevention to avoid recurrence. It is a fact that patients at the highest risk of fracture, those who have already sustained a fracture, have overwhelmingly remain unidentified for osteoporosis treatment and falls prevention. Exceptions exist and they have become examples of best practice; whereby integrated, systematic identification, investigation and intervention were key components for secondary prevention of fractures. The cornerstone in such programs is the fracture coordinator; hence, they are commonly referred to as a fracture liaison service (FLS) They are the link between the orthopaedics and the osteoporosis clinic. Through the development of a best practice framework, the key components for developing secondary fracture prevention programs are outlined and tool kits on how to get started are available. The fundamental component is however, acceptance by the system in order to incorporate prevention as a required part of fracture management.

The presentation will provide an overview of main advances as well as tips on how to move forward.

Disclosure of Interest: K. Åkesson Speakers bureau: Invited lectures for Amgen, Lilly, Radius, UCB DOI: 10.1136/annrheumdis-2018-eular.7834

\section{SP0135 THE EULAR/EFORT RECOMMENDATIONS FOR PATIENTS WITH RECENT FRACTURE}

W.F. Lems, on behalf of Working Group in EULAR EFORt recommendations in patients 50 years and over with a fracture. Rheumatology, location VUmc, Amsterdam Rheumatology and immunolgy Center, Amsterdam, Netherlands

The European League Against Rheumatism (EULAR) and the European Federation of National Associations of Orthopaedics and Traumatology (EFORT) have recognised the importance of optimal acute care for the patient 50 years and over with a recent fragility fracture and the prevention of subsequent fractures in high risk patients, which can be facilitated by close collaboration between orthopaedic surgeons and rheumatologists or other metabolic bone experts. Therefore, the aim was to establish for the first time collaborative recommendations for these patients.

According to the EULAR standard operating procedures for the elaboration and implementation of evidence-based recommendations, 8 rheumatologists from 8 